Artículo

\title{
Desarrollo tecnológico para la optimización de la extracción de nuez pecanera
}

\author{
Humberto Morales Magallanes ${ }^{\S}$ \\ César Landeros Soriano \\ Héctor Villegas-Berúmen
}

Teconologico Nacional de México/ITS Jerez. Libramiento Fresnillo-Tepetongo s/n, Fracc. Los Cardos, Jerez de García Salinas, Zacatecas, México. CP. 99363. Tel. 494 9451274. (landerossoriano@gmail.com; hvillegas@tecjerez.edu.mx).

${ }^{\S}$ Autor para correspondencia: ingmorales2m@gmail.com.

\section{Resumen}

El presente tiene como objetivo mostrar los resultados derivados de una propuesta para la optimización del proceso de extracción de la nuez (Carya illinoinensis Koch), implementando el uso del desarrollo tecnológico como parte del proceso. Lo anterior, en búsqueda de nuevas herramientas tecnológicas para productores de este fruto y así coadyuvar al desarrollo económico a través la disminución de costos, aumento de la eficiencia y mayor producción mediante la optimización de procesos. Esta investigación selecciona un productor local de la comunidad rural 'Lo de Nava', Jerez de García Salinas, Zacatecas. Para la realización del proceso de extracción se analizaron 100 muestras de nuez, de las cuales a 45 se aplicó el proceso de extracción de manera manual y a 55 restantes un proceso optimizado para la separación de la almendra, para recabar los datos de análisis estadístico y conocer los resultados al planteamiento de la investigación. La propuesta para atender la problemática expuesta es el desarrollo de un proceso mecanizado cuyo objetivo consiste en la separación adecuada de la almendra y la cáscara, derivado de un análisis de esfuerzos internos estructurales que faciliten la obtención del fruto, el aumento de la producción y mejoras en su comercialización. El método estadístico utilizado para el análisis de datos es Chi cuadrado $\left(\mathrm{X}_{\text {calculada }}^{2}=8.13\right.$, la cual es mayor a $\left.\mathrm{X}_{\text {critica }}^{2}=3.841\right)$, con un nivel de significancia $(\alpha=$ 0.05), la decisión se encuentra en la zona de rechazo para $\mathrm{H}_{0}$; satisfaciendo la aceptación de la hipótesis alternativa $\mathrm{H}_{1}$. Los resultados muestran que la implementación del desarrollo tecnológico en el proceso de extracción de nuez depende del método utilizado para dicho proceso, ya que se tiene mayor extracción del fruto entero en menor tiempo y mayor calidad en relación con los procesos revisados; que refleja aumento de ventas, al contar con una ventaja competitiva que fomente el desarrollo de los productores. Se concluye que las empresas deben orientarse a la innovación de sus procesos mediante el desarrollo tecnológico, los cuales son de importancia para la región donde se realizó el análisis; así mismo, se debe considerar las técnicas que aporten un análisis para detectar sus necesidades, centralizar los esfuerzos y obtener un resultado eficiente.

Palabras clave: Carya illinoinensis, desarrollo tecnológico, optimización, proceso, producción.

Recibido: mayo de 2020

Aceptado: julio de 2020 


\section{Introducción}

Según Díaz (2008), el nogal pecanero, productor de la nuez Carya illinoinensis Koch, comprende plantas arbóreas que producen un fruto, en la cual durante la fase de maduración el pericarpio y el mesocarpio se secan y el endocarpio (cáscara) y la semilla (almendra) son consideradas una nuez. La nuez pecanera tiene un sabor mucho más agradable que las demás y se caracteriza por sus diferentes usos en pastelerías, panaderías, bisqueterías, confiterías, neverías y dulcerías, ya que cuenta con un alto valor nutritivo.

La nuez pecanera es una fruta seca, apreciada y consumida en todo el mundo por su sabor particularmente agradable y su riqueza en componentes saludables. La producción mundial de nuez pecanera la encabezan Estados Unidos de América y México, el primero cuenta con 55\% de producción mundial y el segundo con 38\%, los países que contribuyen con el resto son Sudáfrica, Australia y otros (International Nut y Dried Fruit, 2014). Este fruto se cultiva principalmente el este y sur de Estados Unidos de América, siendo los principales productores los estados de Georgia, Texas, Nuevo México, Arizona, Luisiana y Oklahoma (USDA-NASS, 2011). Los españoles llamaron nogal al árbol pecanero y a su fruto, la pecanera, la nombraron 'nuez'. En distintas regiones del país se le diferencia de otras nueces con el nombre de nuez 'cáscara de papel' (Toole, 1965; Brison, 1976).

La introducción pionera de plantaciones comerciales en México se hizo en 1904, en el estado de Nuevo León. En el estado de Chihuahua las primeras huertas comerciales se establecieron en el año de 1946, mientras que en la Comarca Lagunera se inició la explotación del nogal en 1948. A partir de esos años, la superficie plantada con nogales se ha incrementado notablemente en toda la región norte del país. Para 1991 México aportaba 15\% de la producción mundial de nuez, ocupando el segundo lugar en producción (INEGI, 1991). Actualmente, los estados mexicanos más destacados en producción de nuez son Chihuahua, Sonora, Coahuila y Durango con participaciones relativas de $63.14 \%, 13.39 \%, 10.83 \%$ y $5.74 \%$, respectivamente (SIAP, 2018).

De acuerdo con las características orográficas, tipo de suelo y clima del municipio de Jerez de García Salinas, se puede considerar que se cuenta con los elementos para el buen desarrollo del cultivo de nuez pecanera. De manera empírica, se identifica que, en la región de Jerez de García Salinas, Zacatecas, la temporada de producción de la nuez inicia a principios de octubre y se concentra en el mes de noviembre, cuando los árboles alcanzan su máxima producción, la cual puede extenderse hasta el mes de diciembre, dependiendo de las condiciones climáticas, ya que las heladas y el viento aceleran la caída natural del fruto, la cual es también forzada por medio de vibración aplicada artificialmente a los árboles.

Según Orona (2013), en el estado de Zacatecas el nogal es de gran importancia, ya que la región norte del estado forma parte del centro de origen de esta especie. La producción de nuez en México a nivel de entidades federativas para el año 2010 se presenta en el Cuadro 1. De acuerdo con Gerónimo (2011), la producción es el proceso de creación de los bienes y servicios que la población puede adquirir para consumirlo y satisfacer sus necesidades, comúnmente este proceso se lleva a cabo en empresas, las cuales se encuentran integradas en ramas productivas y éstas en sectores económicos. 
Cuadro 1. Producción de nuez en México, 2010.

\begin{tabular}{cccc}
\hline Estados & Superficie cosechada (ha) & Producción $(\mathrm{t})$ & ${\text { Rendimiento }\left(\mathrm{t} \mathrm{ha}^{-1}\right)}^{\text {Aguascalientes }}$ \\
Coahuila & 177 & 342.4 & 1.93 \\
Chihuahua & 12910.9 & 10247.5 & 0.79 \\
Durango & 39420.6 & 39764.9 & 1.01 \\
Guanajuato & 4069.8 & 3652.1 & 0.9 \\
Hidalgo & 86 & 92.6 & 1.08 \\
Nuevo León & 738.7 & 2389.2 & 3.23 \\
San Luis Potosí & 3807.3 & 1679 & 0.44 \\
Sonora & 122 & 466 & 3.85 \\
Tamaulipas & 7437.5 & 16102.9 & 2.16 \\
Zacatecas & 89.5 & 165.6 & 1.85 \\
Total nacional & 43 & 69.8 & 1.62 \\
\hline
\end{tabular}

En la agricultura, como en otras actividades económicas, constantemente están surgiendo desarrollos tecnológicos que permiten incrementar la productividad. Una manera de hacer llegar esos desarrollos a los agricultores es la capacitación. De acuerdo con Nakano et al. (2018), la capacitación es un método efectivo para difundir las nuevas tecnologías, incrementar la productividad y disminuir la pobreza rural. Dado que la capacitación tiene un alto costo, el reto para estos autores es la elección del mejor método al más bajo costo para llevar los conocimientos a los agricultores (Nakano et al., 2018).

Cabe mencionar, que dichas empresas actúan como generadoras de innovaciones en sus procesos y productos, ya que esto les proporciona ventajas competitivas. Para desarrollar la innovación, una compañía debe antes que nada buscar, identificar y evaluar conocimientos alternativos de diferentes fuentes y posteriormente, identificar el conocimiento potencialmente útil. La empresa debe transferir luego ese conocimiento, a partir de las fuentes disponibles y buscar que este permee el conjunto de la organización (Zhou y Wu, 2010).

Según Carbajal (2010), las capacidades tecnológicas implican la oportunidad de potenciar las oportunidades y las posibilidades del desarrollo de la innovación tecnológica, con la intención de fortalecer las capacidades individuales y colectivas para fomentar lo que las personas y las sociedades quieren y pueden ser. El desarrollo de la innovación tecnológica, si bien a veces depende de las destrezas individuales, requiere de un contexto cultural y político.

Este contexto involucra al colectivo, los grupos o las sociedades, los que han de contar con un mínimo de condiciones para lograr el desarrollo tecnológico. Este mínimo de condiciones tiene que ver con las capacidades tecnológicas que han de estar presentes en el colectivo para poder realizar lo que las comunidades quieren hacer y ser. Esta ampliación de las nociones de capacidades y funcionamientos de lo individual a lo colectivo y al ámbito de lo tecnológico se realiza a partir de un procedimiento por analogía en el que se comparan las características de tales ideas a la de capacidades tecnológicas. 
Por ejemplo, Porter (1980) refiere a la capacidad no en tanto posibilidad de hacer, sino en tanto incremento de actividades, productos y productividad. Idea que remite a la metáfora del recipiente: el incremento de la capacidad es cómo agregar capacidad para apoyar los objetivos de la empresa o su participación en el mercado, en tanto se evita la sobrecapacidad del sector industrial.

También concibe la capacidad en tanto el tamaño de las adiciones, como si la capacidad fuese el aumento de tamaño y la producción: la tecnología futura es importante porque es necesario pronosticar la probabilidad de que las actuales adiciones a la capacidad queden obsoletas o que los cambios en el diseño permitan incrementos efectivos en la capacidad en el sitio actual de las instalaciones.

Zhou y Wu (2010) han identificado los beneficios más comunes de las capacidades tecnológicas. En orden de importancia se encuentran la posibilidad de diseñar una estrategia adecuada e integral, la flexibilidad para la innovación de procesos y productos y la identificación de en dónde asignar los recursos disponibles Combs et al. (2011), toda vez que las empresas dependen cada vez más del uso óptimo de estos para poder innovar.

En consonancia con ello, Benner y Tushman (2003) refieren que las empresas deben invertir de manera decidida en la construcción de capacidades tecnológicas. Esto para desarrollar destrezas y habilidades a fin de utilizar diversos recursos adecuadamente y saber cómo hacer las cosas. Hoy se considera que, en la medida en que las empresas acumulan experiencias, conocimientos y autoaprendizaje, logran aprovechar más sus habilidades.

Según Tartabull (2015), un papel esencial en el desarrollo tecnológico les corresponde a las universidades y a los centros tecnológicos. Al respecto Barge et al. (2011) refieren que en la mayoría de los países desarrollados los centros tecnológicos se consideran un importante elemento de la infraestructura tecnológica nacional y regional, representan un imprescindible eslabón en la prestación de servicios a las empresas, abarca desde la investigación aplicada y el desarrollo tecnológico hasta otros servicios de apoyo, como por ejemplo la consultoría, el diagnóstico y la asistencia técnica.

Por otra parte se puede considerar que la ciencia y el desarrollo tecnológico es mutuo entre empresas e instituciones educativas; por lo tanto, para Tartabull (2015), un elemento base para lograr el desarrollo está centrado en el empleo de la ciencia y la tecnología, materializado mediante los procesos de innovación que a diferentes niveles se ejecuten, es fundamental el establecimiento que genere alianzas estratégicas, sobre todo entre las universidades y el sector empresarial, Othman (2011) lo cataloga como una cooperación que da beneficio para ambas partes, principalmente, si se analiza que una aporta soluciones y la otra escenarios para la formación de capacidades que no se pueden lograr desde el desempeño de la universidad intramuros, proceso que redundará nuevamente en un beneficio para la industria, como fuente de insumos de la mayor parte de los graduados de las diferentes disciplinas, de aquí la necesidad de programas formativos que tomen en cuenta las demandas de las industrias, esto genera capacidades que apuntan la sostenibilidad del desarrollo desde múltiples aristas y cualquiera de ellas muy interesante en su análisis particular.

Para Aström et al. (2008) un centro de desarrollo tecnológico (CDT) es aquella organización que esparce conocimiento adquirido mediante sus propios procesos de investigación, dirigido a que sus clientes lo pongan en práctica dentro de sus propias operaciones. Se presentan como socios 
estratégicos de las empresas con la finalidad de lograr un mayor desarrollo y fortalecimiento de su status innovador y competitivo. Por su parte, Barge-Gil y Mondrego (2007), consideran como cualidad de un CDT su dedicación a la prestación de servicios de innovación y desarrollo tecnológico para las empresas y los sectores industriales.

Para Valencia (2019), la transferencia tecnología es posible bajo el esquema de investigación, desarrollo e innovación (I+D+i), proceso en el cual los CDT forman parte del proceso de transmitir el saber-hacer entre los distintos sectores. El conocimiento emanado de los CDT permite lograr altos niveles de competitividad y desarrolla la innovación producto de la inversión que transforma el conocimiento en dinero proveniente de la satisfacción de las diversas necesidades del mercado. Por lo tanto, los CDT son estructuras especializadas en la realización de tareas para la generación de valor agregado a distintos productos o servicios en determinadas industrias.

Mas-Verdú (2008) reconoce el rol de los CDT y las universidades, que en conjunto permiten generar la infraestructura necesaria para la innovación, siendo prestadoras de servicios dirigidos al impulso de varias fases de la actividad innovadora de las empresas, principalmente en la generación y adquisición de conocimiento y tecnología, así como en la preparación para la producción y la comercialización.

Según Orona (2007), la comercialización de la nuez es un aspecto igual de importante que la producción y en este sentido se identificó que $88 \%$ de los productores venden su producto en forma embalada (directa), esto es, sin agregación de valor. El 12\% restante vende parte de su producción sin cáscara, logrando precios tres veces superiores a los que reciben al vender en forma directa.

El precio se determina en el mercado de los Estados Unidos de América de acuerdo con las fuerzas de la oferta y la demanda, así como por su calidad determinada por el tamaño y color de la almendra (Espinoza et al., 2009). Ojeda et al. (2010), señala que se requiere instrumentar estrategias que permitan darle valor agregado a la nuez realizando estudios de mercados para la nuez, productos derivados y de buscar alternativas de comercialización. Además, las fluctuaciones que tiene el precio de la nuez impactan la demanda de insumos. El cultivo del nogal en México es de interés ya que es el segundo productor mundial de este producto.

Rostow (1973) indica que el crecimiento económico atraviesa por cinco etapas (sociedad tradicional, condiciones previas al impulso inicial, el impulso inicial o despegue, la marcha hacia la madurez y la era del alto consumo masivo), las condiciones específicas tales como propensión al ahorro y la inversión y el espíritu de iniciativa, generan disposición al adelanto tecnológico para poder pasar de sociedades tradicionales a sociedades de consumo masivo.

Para Rostow (1973), la etapa de despegue incluye un rápido crecimiento de ciertos sectores guías, con modernas técnicas industriales que permiten difundir el desarrollo tecnológico. Su planteamiento encaja con el impulso de centros de desarrollo tecnológico, pues busca incorporar procedimientos de producción avanzados. La crítica a su planteamiento radica en que dichas etapas son imaginarias, pues los países avanzados no han registrado un solo camino para lograr el crecimiento económico, sino que muchas veces no atravesaron dichos momentos para lograr sus avanzados niveles de desarrollo. 
El objetivo de este trabajo consiste en analizar la relación que existe en la optimización de proceso, mediante la implementación del desarrollo tecnológico en el proceso de la extracción de la nuez, adecuando las necesidades que tienen las empresas en cuanto a la existencia de procesos innovadores que facilitan su crecimiento y funcionalidad, todo este proceso se obtiene realizando un análisis estadístico prueba de Chi Cuadrado que permita relacionar las variables con datos extraídos y dar una validez a la hipótesis planteada de los datos analizados.

\section{Materiales y métodos}

\section{Localización del estudio}

El estudio se realizó en una huerta de nogal en producción de 20 años, con una densidad de 100 árboles ha $^{-1}$, establecida dentro la comunidad Lo de Nava se localiza en el Municipio Jerez de García Salinas del estado de Zacatecas, México y se encuentra en las coordenadas GPS, longitud (dec): - 102.926389 y latitud (dec): 22.73250, se encuentra a una mediana altura de $2080 \mathrm{msnm}$.

Con la información obtenida, de cada nuez sobre el proceso de extracción en la cual se va a evaluar la correlación entre la implementación del desarrollo tecnológico para la optimización del proceso de extracción y el método de extracción manual de la nuez, para obtener la función de producción adecuada. Con este objeto se toma una muestra de 100 nueces las cuales se clasifica en la Cuadro 2 de asociación, encontrando los siguientes resultados:

Cuadro 2. Asociación y valores observados.

\begin{tabular}{cccc}
\hline $\begin{array}{c}\text { Tipo de método de } \\
\text { extracción de nuez }\end{array}$ & $\begin{array}{c}\text { Extracción } \\
\text { manual }\end{array}$ & $\begin{array}{c}\text { Desarrollo tecnológico para la } \\
\text { optimización del proceso de extracción }\end{array}$ & Total \\
\hline Extracción en trozos & 20 & 25 & 45 \\
Extracción entera & 10 & 45 & 55 \\
Total & 30 & 70 & 100 \\
\hline
\end{tabular}

Los datos se analizaron con un modelo estadístico de Chi-Cuadrado. Donde los factores fueron: 1) tipo de métodos de extracción (extracción manual $v s$ desarrollo tecnológico); y 2) optimización para la obtención del fruto (trozos vs entera) conforme del tamaño del producto de la huerta. Por lo anterior se formula lo siguiente: ¿Permiten estos datos afirmar que el uso desarrollo tecnológico es independiente del método de extracción? Se usará un nivel de significación alfa $=0.05$. El análisis de estos valores se encuentra determinados en la Cuadro 3 obteniendo los valores esperados los que permiten calcular la prueba estadística en relación del valor de tabla de distribución de frecuencias, que se notan en cursiva, para su adecuada identificación.

Cuadro 3. Asociación y valores esperados.

\begin{tabular}{cccc}
\hline $\begin{array}{c}\text { Tipo de método de } \\
\text { extracción de nuez }\end{array}$ & $\begin{array}{c}\text { Extracción } \\
\text { manual }\end{array}$ & $\begin{array}{c}\text { Desarrollo tecnológico para la } \\
\text { optimización del proceso de extracción }\end{array}$ & Total \\
\hline Extracción en trozos & 13.5 & 31.5 & 45 \\
Extracción entera & 16.5 & 38.5 & 55 \\
Total & 30 & 70 & 100 \\
\hline
\end{tabular}


De los datos tabulados se identifican los valores obtenidos, los cuales representan la proporción alcanzada de cada uno de los métodos de extracción, por lo que al realizar los análisis en el método estadístico Chi cuadrado, arrojan los siguientes resultados: $X^{2}$ calculada $=8.1289$, la cual es mayor a $\mathrm{X}_{\text {crítica }}=3.841$, por lo que la decisión se encuentra en la zona de rechazo para H0 como lo muestra la Figura 1.

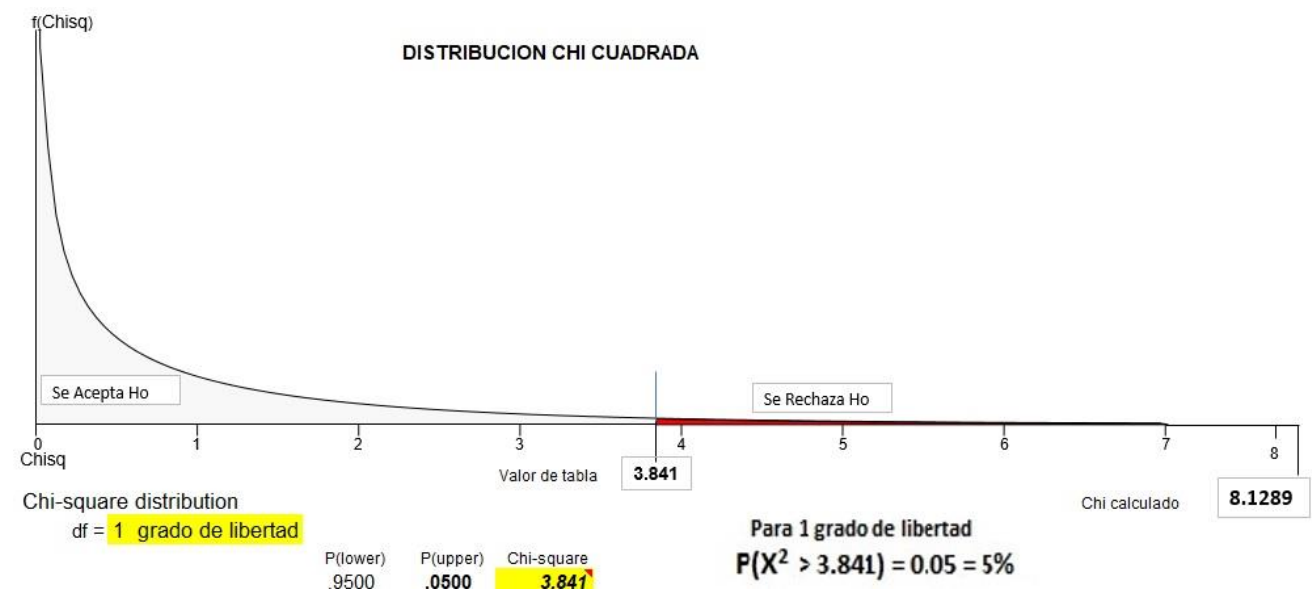

Figura 1. Distribución de Chi cuadrada para obtener grado de rechazo de $\mathbf{H}_{0}$.

Por lo anterior, se acepta la hipótesis alternativa, que plantea que las variables de la implementación del desarrollo tecnológico para la optimización del proceso y el método de extracción es dependiente, considerando esto para la mejor extracción de la nuez entera, esto da un mayor margen para obtener altas producciones de mejor calidad en relación al valor de venta del fruto, por lo que la optimización del proceso de extracción de la nuez es fundamental para la adecuación del desarrollo tecnológico dentro del área agroindustrial.

\section{Resultados y discusión}

Conforme a la obtención de los datos recolectados, arrojados de las pruebas realizadas, se determinan las variables a analizar, como el método de extracción y la optimización del proceso de extracción, estas son factores determinantes dentro del análisis y los aspectos como la eficiencia, la mejora del proceso de extracción de manera entera de la nuez, el procedimiento para la mejor comercialización del fruto, la infraestructura adecuada y el equipamiento optimizado para el proceso, entre otras características.

A continuación, se presentan los resultados del análisis para el productor 'Lo de Nava' localizado en el Municipio Jerez de García Salinas del Estado de Zacatecas. De los datos analizados a las 100 piezas de nuez que se tomaron como muestra para la aplicación de un esfuerzo estructural, 30 de ellas se seleccionaron para el método de extracción manual, de las cuales 20 se extrajeron en trozos y 10 de manera entera, obteniendo una optimización de 33\% de eficiencia en producto entero; por otro lado, de las 70 unidades en que se utilizó el desarrollo tecnológico, 25 se extrajeron en trozos y 45 de manera entera, por lo cual, se tiene 64\% de eficiencia en la extracción del fruto entero. 
Derivado de los datos obtenidos y del contraste de hipótesis por el método estadístico de prueba de Chi Cuadrado, resultando un valor $\mathrm{X}_{\text {calculada o prueba }}^{2}=8.1289$, la cual es mayor al valor $\mathrm{X}^{2}$ crítica o tabla $=3.841$, la cual tuvo como finalidad someter los datos obtenidos a prueba hipótesis referidas a distribuciones de frecuencias que permiten determinar que la hipótesis nula (Ho) se rechaza y la hipótesis alternativa (H1) se acepta indicando que las variables de la implementación del desarrollo tecnológico para la optimización del proceso y el método de extracción es dependiente, por lo que la utilización de nueva maquinaria y equipo ayuda para la mejor extracción de la nuez entera.

En contraste con lo encontrado, el método de extracción manual se observa una eficiencia en la extracción de la nuez entera de $33 \%$ y el método del desarrollo tecnológico utilizado en la optimización de la extracción de la nuez se obtuvo 64\% de eficiencia en la extracción del fruto entero, los resultados coinciden con Carbajal (2010), Las capacidades tecnológicas implican la oportunidad de potenciar las oportunidades y las posibilidades del desarrollo de la innovación tecnológica, con la intención de fortalecer las capacidades individuales y colectivas para fomentar lo que las personas y las sociedades quieren y pueden ser.

Derivado de la importancia de adecuar las necesidades de equipamiento e infraestructura que permita recolectar y procesar el fruto, se realiza un análisis de la infraestructura del productor con el objetivo de aprovechar los recursos disponibles de que les facilite la innovación y producción de la nuez, la tendencia que describe Zhou y Wu (2010) han identificado los beneficios más comunes de las capacidades tecnológicas. En orden de importancia se encuentran la posibilidad de diseñar una estrategia adecuada e integral, la flexibilidad para la innovación de procesos y productos y la identificación de en dónde asignar los recursos disponibles.

Los productores de la localidad 'Lo de Nava' deben consolidarse como una asociación productora de nuez, que inviertan capital además de bajar fondos gubernamentales para apoyo de infraestructura y equipamiento, coincido con Benner y Tushman (2003) refieren que las empresas deben invertir de manera decidida en la construcción de capacidades tecnológicas.

Al contar con un proceso optimizado que permita la producción de nuez entrera permite a los productores de este fruto obtener mejor calidad, producción y distribución del producto para su comercialización a un mejor precio dentro del mercado nacional e internacional, según Orona (2013). Comercialización. En lo referente a los canales de comercialización, en los productores del estrato 1 predomina la comercialización a granel sin selección previa por no contar con suficientes recursos para realizar otras formas de comercialización, en el estrato 2, es equilibrada la forma de comercializar a granel sin selección previa y a granel seleccionada por tamaño y en el estrato 3 la forma de comercialización predominante es a granel seleccionada por tamaño, en la cual estos productores cuentan con mejor infraestructura y recursos para realizar la selección de la nuez.

\section{Conclusiones}

Los productores de nuez de la zona norte del país tienen formas de extracción de nuez más avanzados que los del municipio de Jerez de Gracia Salinas, Zacatecas, debido a que en este lugar existen productores que solo comercializan sin agregar valor a su producto y además, sin recurrir a la utilización del desarrollo tecnológico como herramienta de optimización del proceso de la extracción del fruto y por ende, reciben precios más bajos por su producto debido a que sus ventas 
del producto son a granel por no contar con la tecnología y proceso adecuado para el máximo aprovechamiento del producto ya procesado, e incluso, al no contar con una marca propia reconocida que los identifique como empresas que cuentan con un proceso de extracción optimo, de calidad y eficiente, que dé variedad dentro del tipo de método de extracción para una mejor comercialización de su producto y derivados.

Esta situación depende de la visión y asesoría que los productores reciban tanto en procesos adecuados que sean simplificados y de un diseño y planificación eficiente en la instalación de equipos con capacidad de producción que permita el paso al desarrollo tecnológico, por lo que es necesario contar con la capacidad tecnológica adecuada, tanto en infraestructura y equipo tecnológico que permita el desarrollo de las empresas, siendo la innovación una herramienta que de acceso a la optimización de los procesos dentro de cualquier industria que tienda al crecimiento tecnológico.

En este caso, la recomendación es fortalecer, adiestrar e impulsar el desarrollo tecnológico de las empresas mediante la innovación de procesos tecnológicos, maquinaria y equipo que ayuden a facilitar los procesos y además de la asesoría de empresas similares que ya tengan en marcha este proceso y así fortalecer el crecimiento industrial y económico del estado, con mayor capacitación e integración no sólo en lo productivo sino en la industrialización del producto por medio de maquinaria que incremente la productividad en la etapa de extracción del fruto.

La adopción de este tipo de equipamiento permite la optimización del proceso de extracción de nuez, por lo anterior el desarrollo tecnológico aplicado dentro de la empresa aumenta la productividad, así se incrementa la eficiencia dentro del proceso para la extracción de nuez entera, y así se fortalece la comercialización del producto, que es lo que los empresarios pretenden para así exportar su producto por diferentes canales de distribución de manera nacional, así como internacional.

\section{Agradecimientos}

A la empresa 'Lo de Nava' por la disponibilidad para la realización del análisis de pruebas a las muestras de nuez dentro de sus instalaciones, al laboratorio de investigación, y al laboratorio de metal mecánica del Instituto Tecnológico Superior de Jerez, por su capacitación, adiestramiento y facilidad para el uso de herramientas y equipos. De igual forma al cuerpo académico reconocido ante PRODEP ‘optimización de procesos y ciencia de datos' ITSJ-CA-1.

\section{Literatura citada}

Aström, T.; Eriksson, M.L. y Arnold, E. 2008. International comparison of five institute systems. copenhagen: Forsknings-og Innovationsstyrelsen. 17 p.

Barge, A.; Santamaría, L. and Modrego, A. 2011. Complementarities Between Universities and Technology. New Empirical Lessons. European Planning Studies. 19(2):195-215.

Barge-Gil, A. y Modrego-Rico, A. 2007. "Los centros tecnológicos como instrumentos de intervención pública”. En: X. Vence (editor), crecimiento y políticas de innovación: nuevas tendencias y experiencias comparadas. Madrid: Ed. Pirámide. 241-271 pp. 
Benner, M., \& Tushman, M. (2003). Exploitation, exploration and process management: The productivity dilemma revisited. Academy of Management Review. 28(2):238-256.

Brison, R. F. 1976. Cultivo del nogal pecanero. $1^{a}$ edición en español. Editorial CONAFRUT. México. $350 \mathrm{p}$.

Carbajal-Villaplana, Á. 2010. Las capacidades tecnológicas como base para el desarrollo. Revista electrónica "Actualidades Investigativas en Educación". http://www.redalyc.org/ articulo.oa?id=447/44713068010.

Combs, J. G.; Ketchen Jr, D. J.; Ireland, R. D. and Webb, J. W. (2011). The role of resource flexibility in leveraging strategic resources. J. Manag. Stud. 48(5):1098-1125.

Diaz Morales, M. 2008. Canales y márgenes de comercialización de la nuez pecanera en el municipio de Parras de la Fuente, Coahuila. UAAAN. Tesis presentada en UAAAN. 3 p.

Espinoza, A. J. J.; Salinas, G. H.; Orona, C. I. y Palomo, R. M. 2009. Planeación de la investigación del INIFAP en la Comarca Lagunera en base a la situación de mercado de los principales productos agrícolas de la región. Cuarta Época. Rev. Mex. Agron. 24(13):758-773.

Gerónimo, Z. J. 2011. Teoría de la producción y costos. https://moncedy.files.wordpress.com/ 2011/12teoria-de-laproduccic3b3n-y-costos-unidad-4-econimia-penultima-unidad.pdf.

INEGI. 1991. El nogal en cultivos perennes de México. VII censo agropecuario. Primera edición, impreso en México. 225 p.

International Nut and Dried Fruit. 2014. Global statistical review 2014-2015. https://www.nutfruit.org/wp-continguts/uploads/2015/11/global-statistical-review-20142015_101779.pdf.

Mas-Verdú, F. 2008. "Does only size matter in the use of knowledge intensive services?" Small Business Economic. 31:137-146.

Nakano, Y.; Tsusaka, T.; Aida, T. and Pede, V. 2018. Is farmer-to-farmer extension effective? The impact of training on technology adoption and rice farming productivity in Tanzania. World Development. 105(2018):336-351.

Ojeda, B. D. L.; Arras, V. A. M.; Hernández, R. A.; López, D J. C.; Aguilar, V. A. y Denogean, B. F. G. 2010. Análisis FODA y perspectivas del cultivo del nogal pecanero en Chihuahua. Rev. Mex. Agron. 14(27):357.

Orona-Castillo, I.; Espinoza-Arellano, J.; González-Cervantes, G.; Murillo-Amador, B.; GarcíaHernández, J.; y Santamaría-César, J. 2007. Aspectos técnicos y socioeconómicos de la producción de nuez (Carya illinoensis) en la Comarca Lagunera. Revista Mexicana de Agronegocios. Xl(20):299-307.

Orona, C. I.; Sangerman, J. D. M.; Fortis, H. M.; Vázquez, V. C. y Gallegos, R. M. A. 2013. Producción y comercialización de nuez pecanera (Carya illinoensis Koch) en el norte de Coahuila, México. Rev. Mex. de Cienc. Agríco. 4(3):461-473.

Othman, N. 2011. An Assessment of a University-Industry Partnership in a Malaysian University. Revista International Journal of Business and Social Science. 2(8):94-103.

Porter, M. 1980. Ventaja competitiva. Creación y sostenimiento de un desempeño superior. México, D. F. Compañía Editorial Internacional.

Rostow, W. W. 1973. The Stage of Economic Growth. The Economic History Review. 12 (1):1-16.

SIAP. 2018. Sistema de Información Agroalimentaria y Pesquera. Servicio de Información Estadística Agroalimentaria y Pesquera.

Tartabull-Contreras, Y.; Rivero-Casanova, C. J. y Briones-Kusactay, V. H. 2015. El desarrollo tecnológico, la sostenibilidad, la gestión del conocimiento y el desarrollo social. Revista Universidad y Sociedad. 7(3):74-78 pp. http://rus.ucf.edu.cu/. 
Toole, E. R. 1965. Pecan (Carya illinoensis). In: Fowells, H. A. (Comp.). Silvics of forest trees of the United States. Agriculture Handbook 271. Washington, DC. 12-15 p.

USDA-NASS. 2011. Annual non citrus fruits and nuts summary. 1(2).

Valencia, L. O.; Soto, H. D. \& Cruz, M. C. 2019. Centros de desarrollo tecnológico en México: teoría, contextos, innovación e implicaciones. Revista Latinoamericana de Desarrollo Económico. 17(31):161-180. http://www.scielo.org.bo/scielo.php?script=sci_arttext\&pid $=$ S2074-47062019000100007 \&lng=es\&tlng=es.

Zhou, K. Z. and Wu, F. (2010). Technological capability, strategic flexibility and product innovation. Strategic Management Journal. 31(5):547-561. 